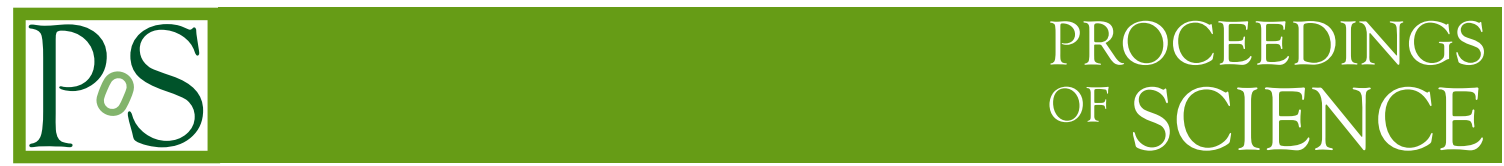

\title{
HERAFitter - an open source QCD fit framework
}

\author{
Andrey Sapronov* \\ on behalf of the HERAF itter team \\ JINR (Joint Institute for Nuclear Research, Dubna) \\ E-mail: sapronov@cern.ch
}

The presented QCD analysis framework, HERAF itter, is an open source PDF fitting tool developed for the extraction of PDFs from the experimental results and for the evaluation of impact of new data. The framework includes dedicated modules for various theoretical and methodological options, as well as techniques to account for various sources of uncertainties. It is capable to fit a large number of relevant data sets finding its application in QCD analyses based on data obtained at HERA, Tevatron and the LHC experiments.

The European Physical Society Conference on High Energy Physics -EPS-HEP2013

18-24 July 2013

Stockholm, Sweden

* Speaker. 


\section{Introduction}

The parton distribution functions (PDFs) is a research field spurring many of the QCD analyses of the experimental high energy physics data. While the hadronic interactions cannot be duly described using perturbative approximation, the factorization theorem helps isolating the cross section components which can be determined empirically. This procedure have to be properly performed for higher order approximations with possible inclusion of collinear divergences of partonic cross sections into the partonic distributions.

The PDF extraction procedure in QCD analysis implies fitting the experimental data with theoretical predictions based on parametrized partonic distributions at a starting scale which are then evolved to the scale of the measurements using DGLAP [8] evolution equations. The objective is to utilize as much information from precision data as possible, with an important care regarding treatment of the uncertainties and accounting for possible correlations in order to improve precision of calculations.

Several groups are currently involved in the PDF fitting analysis: MSTW [1], CT(EQ) [2], NNPDF [3], AB(K)M [4], GJR [5] and HERAPDF [6]. Their methods and distinguishing features are reviewed in [7]. In short, all groups perform global fits of the deep inelastic scattering (DIS), fixed target and $p p(\bar{p})$ collider data, except HERAPDF which relies purely on the HERA data and hence justified usage of the $\chi^{2}$ tolerance of $\Delta \chi^{2}=1$. The NNPDF approach is notable for their analysis being performed via neural network training is association with a Monte Carlo method to estimate their uncertainties and minimise the input parametric form dependence on PDFs. There are other various differences among PDF groups: higher orders counting, heavy flavour corrections, treatment of $\alpha_{S}$, etc. The results are being constantly cross checked against each other at the PDF4LHC working group [9].

The interest of achieving better level of agreement among these PDF groups, as well as an overall better understanding of the proton's PDF is driven by the recent results from the LHC could they be Standard Model (SM) or Beyond SM for which PDFs still represent a dominant source of the systematic uncertainties.

\section{Overview of the HERAF itter program}

HERAFitter is an open source QCD fitting framework with main application for extraction of PDFs from experimental data. The program is designed with a user friendly but highly configurable interface and includes a large number of theoretical models. It allows extensive study of the impact of new experimental data. A detailed information about the project status and available releases with documentation can be found in http://herafitter.org.

The analysis is based on the $\chi^{2}$ estimate of how well the theoretical prediction describe experimental data for a given PDF parametrisation. Various ansatz on PDF parametric form can be tested within this framework. The framework is designed such that a user can plug in his own sets of data with corresponding theory prediction, adjust input PDF parametrisation form, select a specific desired scheme for matching the heavy quark thresholds, a calculation order, cuts, etc. The QCD fit output will provide a quantitative evaluation of level of agreement between data and theory, and 
if desired, the resulting PDF grids can be obtained. The package contains elaborate plotting tools for a prompt inspection of the results.

The overall analysis approach is schematically shown in the figure 1. The procedure starts with reading the input parameters and definition of initial parametrisation at the starting scale $Q_{0}^{2}$. Initial scale is chosen to be below the charm mass threshold, as evolution is performed starting from a three active quarks as required by the QCDNUM [10] program, which performs the QCD evolution. The minimisation of the $\chi^{2}$ is performed via the MINUIT [11] package.

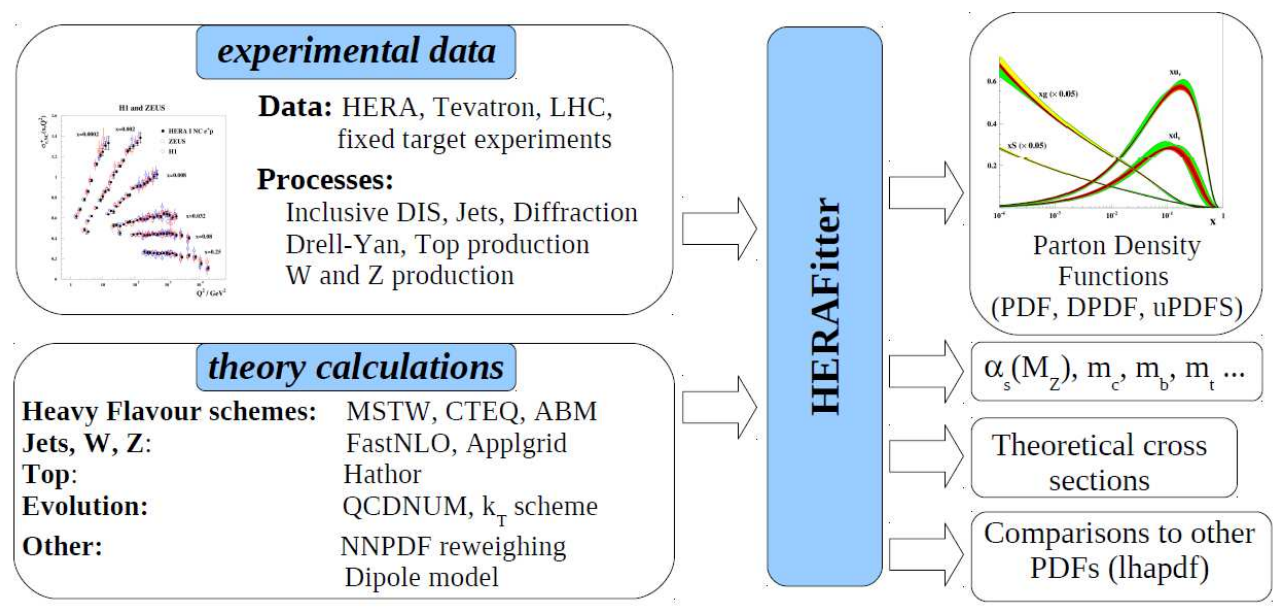

Figure 1: Schematic structure of the HERAF itter program: The "experimental data" block illustrates variety of processes which can be included into the framework and are matched to the corresponding theoretical calculation illustrated in the block below; after the QCD fit is performed the resulting PDFs together with theoretical cross sections are provided as shown on the right side of the figure.

The experimental data are introduced as a formatted text tables along with a header that needs to be adjusted by the user, including e.g. reaction name, data table format and theory parameters. The HERAFitter program features elaborate correlated uncertainty treatment, which can be presented under various representations, i.e covariance matrix to be supplied in additional data file or as a table with nuisance parameters. Various types of uncertainties can be handled using following implemented methods: hessian, Monte Carlo or regularisation, meaning constraining the PDFs in a flexible parametrisation style.

The current version of the HERAFitter framework allows fitting the following types of data, potentially containing PDF relevant information: inclusive cross sections from HERA DIS and fixed target experiments, Drell-Yan, jet production data (ep, $p p$ and $p \bar{p})$ and heavy quark structure functions.

The DIS structure functions may be computed directly in a variety of heavy quark schemes including the fixed-flavour (FFN) and variable flavour number (VFN) schemes. VFN schemes with various treatments for the heavy quark thresholds include the Thorne Roberts (TR) scheme at LO, NLO and NNLO $[12,13]$ as provided by the MSTW group, the ACOT scheme at LO and NLO as provided by the CTEQ group. The QCDNUM also provides the calculations of the DIS structure functions in the zero-mass VFN and FFN schemes. The FFN scheme is alternatively available via the OPENQCDRAD [14] interface in which the running mass definition [15] is implemented.

Another methods of providing theoretical predictions are the interfaces to tools for fast cross 
section estimation, such as APPLGrid [16] or FastNLO [17]. The calculation of the differential (or total) cross section is performed by convoluting the parametrised PDFs with pregenerated tables of higher order coefficients (NLO or NNLO). Further corrections may be applied via K-factor tables, for example NNLO QCD, computed with FEWZ [18] or NLO EW obtained from SANC [19]. Drell-Yan, jet production in $p p, p \bar{p}$ collisions are the processes for which the fast cross section estimates are applicable. The HATHOR [20] program interfaced in the HERAFitter can be used to calculate approximate total cross section of $t \bar{t}$ production in $p p, p \bar{p}$ collisions.

Various forms of $\chi^{2}$ can be chosen in the HERAF itter for the minimisation procedure which are based on the use of nuisance parameters or on the full covariance matrix. For a single data set, the $\chi^{2}$ function is defined in a form

$$
\chi^{2}=\sum_{i} \frac{\left[m^{i}-\sum_{j} \gamma_{j}^{i} m^{i} b_{j}-\mu^{i}\right]^{2}}{\left(\delta_{i, \mathrm{stat}} m^{i}\right)^{2}+\left(\delta_{i, \text { uncor }} m^{i}\right)^{2}}+\sum_{j} b_{j}^{2}
$$

Here $\mu^{i}$ is the measured central value at a point $i$ with relative statistical $\delta_{i, s t a t}$ and relative uncorrelated systematic uncertainty $\delta_{i, u n c}$, the quantity $m^{i}$ is the theoretical prediction, $\gamma_{j}^{i}$ represents relative correlated systematic uncertainties and $b_{j}$ their shifts. In the case of the covariance matrix, the $\chi^{2}$ function takes the form

$$
\chi^{2}=\sum_{i j}\left(m^{i}-\mu^{i}\right) C_{\text {tot } i j}^{-1}\left(m^{j}-\mu^{j}\right),
$$

where the $C_{\mathrm{tot} i j}^{-1}$ is the total covariance matrix given by the sum of the statistical and systematic covariance matrices. In addition, the different approaches can be combined together, e.g. some systematic uncertainties can be treated using the matrix method, others can be treated using nuisance parameters.

\section{Application in QCD analysis of experimental data}

The HERAFitter framework is actively used by the HERA and LHC experiments. At HERA, the results of QCD analyses using HERAFitter are published in the recent combination of charm production measurements in DIS [21] and inclusive H1 measurements at high $Q^{2}$ with longitudinally polarised lepton beams [22]. The HERAFitter framework has been used in the QCD studies of the $Z$ and $W$ cross sections measured in ATLAS to determine the strange quark density of the proton [23]. Another QCD analysis was performed on the inclusive ATLAS jet data at $\sqrt{s}=2.76 \mathrm{TeV}$ compared to $\sqrt{s}=7 \mathrm{TeV}$ [24]. From the theory side, the work is ongoing on updating the ACOT scheme module (in collaboration with CTEQ group members), inclusion of photon PDF in QCDNUM (on former a publication is foreseen). The QCD studies of LHeC data have been also performed with HERAFitter and published in [25].

\section{Summary}

The HERAFitter project provides a mean for QCD interpretation of the SM analyses of experimental data with source code freely available under GPL v3 license. It is designed to be used 
for a robust PDF extraction and to assess the new data impact on the current PDF determination. The package contains all necessary ingredients to study the proton PDFs incorporating a variety of different data processes and theory calculations and is an optimal platform for QCD related benchmarks.

\section{References}

[1] A.D. Martin et al., Eur. Phys. J. C63, 189 (2009), [arXiv: 0901.0002 ]

[2] H.-L. Lai et al., Phys. Rev. D82, 074024 (2010), [arXiv: 1007 .2241]

[3] R.D. Ball et al., Nucl. Phys. B849, 296 (2011), [arXiv:1101.1300, 1012.0836] R.D. Ball et al., [arXiv:1107.2652]

[4] S. Alekhin, et al., Phys. Rev. D81, 014032 (2010), [arXiv: 0908.2766 ]

[5] M. Glück et al., Eur. Phys. J. C53, 355 (2008), [arXiv: 0709 . 0614] P. Jimenez-Delgado and E. Reya, Phys. Rev. D79, 074023 (2009), [arXiv: 0810 . 4274]

[6] F.D. Aaron et al., JHEP 1001, 109 (2010), [arXiv: 0911.0884 ]

[7] A. De Roeck and R.S. Thorne, [arXiv:1103.0555] G. Watt, [arXiv:1106.5788]

[8] V.N. Gribov, L.N. Lipatov, Sov. J. Nucl. Phys. 15, 438, 675 (1972), G. Altarelli, G. Parisi, Nucl. Phys. B 126,298, (1977), G. Curci, W. Furmanski, and R. Petronzio, Nucl. Phys. B 175, 27 (1980), W. Furmanski and R. Petronzio, Nucl. Phys. B 97, 473 (1980), S. Moch, J. Vermaseren, and A. Vogt, Nucl. Phys. B 688, 101 (2004), A. Vogt, S. Moch, and J. Vermaseren, Nucl. Phys. B 691, 129 (2004).

[9] URL https://wiki.terascale.de/index.php?title=PDF4LHC_WIKI.

[10] M. Botje (2010), http://www.nikef.nl/h24/qcdnum/index.html, [arXiv: 1005 .1481].

[11] F. James, M. Roos (CERN). Jul 1975. 38 pp,

[12] R. S. Thorne and R. G. Roberts, Phys. Rev. D 57, 6871 (1998), [hep-ph/ 9709442 ].

[13] R. S. Thorne, Phys. Rev. D 73, 054019 (2006), [hep-ph/ 0601245$].$

[14] S. Alekhin, OPENQCDRAD, a program description and the code are available via: http://www-zeuthen.desy.de/ alekhin/OPENQCDRAD.

[15] S. Alekhin and S. Moch, Phys.Lett. B 699, 345, (2011), [arXiv: 1011.5790 ].

[16] T. Carli et al., Eur.Phys.J. C66 (2010) 503-524, 2010. 33 pp [arXiv: 0911.2985$]$

[17] D. Britzger et al. [arXiv:1208.3641]

[18] R. Gavin et al., Comput.Phys.Commun. 182 (2011) 2388-2403, [arXiv:1011.3540]

[19] D. Bardin et al., JETP Lett. 96 (2012) 285-289, [arXiv: 1207 . 4400 ]

[20] M. Aliev et al., Comput. Phys. Commun. 182, 1034, 2011, [arXiv:1007.1327].

[21] H. Abramowicz et al. [H1 and ZEUS Collaborations], Eur. Phys. J C73 (2013) 2311, [arXiv:1211.1182].

[22] F. Aaron et al. [H1 Collaboration], JHEP 1209 (2012) 061, [arXiv:1206. 700 07]. 
[23] Georges Aad et al. [ATLAS Collaboration], Phys. Rev. Lett. 109 (2012) 012001, [arXiv:1203.4051].

[24] Georges Aad et al. [ATLAS Collaboration], EPJC 732509 (2013), [arXiv: 1304 . 4739].

[25] J. L. Abelleira Fernandez et al. [LHeC Study Group], "A Large Hadron Electron Collider at CERN" J.Phys.G. 39 (2012) 075001, [arXiv: 1206 . 2913]. 